Article

\title{
Clinical and Nutritional Effectiveness of a Nutritional Protocol with Oligomeric Enteral Nutrition in Patients with Oncology Treatment-Related Diarrhea
}

\author{
Alejandro Sanz-Paris ${ }^{1,2, *}$, Javier Martinez-Trufero ${ }^{3} \mathbb{D}$, Julio Lambea-Sorrosal ${ }^{4}$, \\ Fernando Calvo-Gracia ${ }^{5}$ and Raimon Milà-Villarroel ${ }^{6}$ \\ 1 Department of Endocrinology and Nutrition, Miguel Servet Hospital, 50009 Zaragoza, Spain \\ 2 Instituto de Investigación Sanitaria Aragón (IIS Aragon), 50009 Zaragoza, Spain \\ 3 Department of Oncology, Miguel Servet Hospital, 50009 Zaragoza, Spain; jmtrufero@seom.org \\ 4 Department of Oncology, University Clinic Hospital, 50009 Zaragoza, Spain; juliolambea@yahoo.es \\ 5 Department of Endocrinology and Nutrition, University Clinic Hospital, 50009 Zaragoza, Spain; \\ fcalvo@comz.org \\ 6 Group Research on Wellbeing (GRoW), Blanquerna School of Health Sciences-Universitat Ramon Llull, \\ 08025 Barcelona, Spain; raimonmv@blanquerna.url.edu \\ * Correspondence: asanzp@salud.aragon.es; Tel.: +34-651667352
}

Received: 16 April 2020; Accepted: 23 May 2020; Published: 25 May 2020

\begin{abstract}
Background: Poor nutritional status and diarrhea are common complications in cancer patients. (2) Methods: This multicenter, observational, prospective study evaluated the effectiveness of an oligomeric enteral nutrition (OEN) protocol in the improvement of nutritional status and reduction of diarrhea symptoms. Nutritional status was assessed with the Subjective Global Assessment (SGA), Body Mass Index (BMI) and albumin levels. Diarrhea was evaluated by the frequency and consistency of stools (Bristol Stool form scale). (3) Results: After 8 weeks of OEN protocol, the nutritional status improved in $48.3 \%$ of patients, with an increased proportion of patients at risk of malnourishment $(+27.3 \%)$ at the expense of a decrease of moderately $(-19.9 \%)$ and severely $(-7.3 \%)$ malnourished patients $(p<0.001)$. Serum albumin and BMI significantly increased after 8 weeks of OEN treatment $(p<0.005)$. OEN showed a $71.1 \%$ effectiveness in the improvement of stool consistency. The mean number of stools per day significantly decreased from baseline ( 4.17 stools/day) to week 8 (1.42 stools/day; $p=0.0041)$. The nutritional status significantly improved even in those patients with persistent diarrhea. (4) Conclusion: The proposed OEN protocol seemed to be effective in improving the nutritional status, frequency and consistency of stools in patients with oncology treatment-related diarrhea even in persistent cases.
\end{abstract}

Keywords: oncology treatment-related diarrhea; nutritional protocol; oligomeric enteral nutrition

\section{Introduction}

Despite the improvement in patient's prognosis and life expectancy enabled by cancer therapy, oncological patients frequently suffer from associated side effects, being gastrointestinal complications particularly common [1].

Chemotherapy, other systemic treatments, and radiotherapy can induce and exacerbate the inflammation of the gastrointestinal tract mucosa, resulting in taste changes, nausea, constipation and diarrhea [2]. Of particular importance is oncology treatment-related diarrhea (OTRD) due to its effects on hydration and electrolyte balance, which can severely compromise the nutritional status of patients [3]. The frequency of OTRD ranged from 5 to $74 \%$ in randomized clinical trials, becoming a common cause of hospital admissions and being life-threatening in persistent and severe cases [4]. 
Cancer patients are therefore at high risk for malnourishment, with a prevalence that varies widely with the type and stage of tumor, cancer therapy modality and intensity [5]. Previous studies showed that poor nutritional status was associated with a higher rate of hospital admissions, increased hospital length of stay, reduced quality of life and mortality, and lower tolerance to therapy in cancer patients [6]. All these complications add an extra burden to the negative effects that cancer itself has for patients and healthcare systems [7].

Since these complications are progressive, early identification and intervention in patients at risk will minimize the progression towards more severe states that could prevent from continuing cancer therapy and threaten the life of the patient [7]. However, clinical guidelines mostly focus on the pharmacological treatment of diarrhea but do not specifically address the required nutritional support for patients [4-6,8-10]. Depending on the severity and persistence of symptoms, these interventions can range from dietary counselling and food supplements to medical nutrition therapy [11]. Among the latter, oral enteral nutrition can be an advantageous alternative to meet the nutritional demands of patients with OTRD and clinical stability [12].

However, specific guidance on this type of nutritional support is scarce. To address this unmet need, we recently published a nutritional support algorithm for patients with OTRD using oligomeric enteral nutrition (OEN) [13]. OEN is a nutritionally complete formula in which peptides (instead of whole proteins) are the source of nitrogen and fats are mainly provided as medium-chain triglycerides. This readily digestible source of nutrients increases digestibility, thereby facilitating the absorption of nutrients, protecting mucosal integrity, and showing low antigenic effect [14]. We developed a stepwise approach for patients with OTRD that considers the nutritional status (at risk of malnourishment, moderate or severe malnourishment) and the intestinal function (preserved or impaired) of patients. Depending on the severity and persistence of symptoms, the OEN protocol is recommended in different stages of OTRD to improve the nutritional status of patients and prevent diarrhea and dehydration in the long term [13]. In this study, we present for the first time the impact of this protocol of nutritional support in a population of patients with cancer and OTRD.

\section{Materials and Methods}

\subsection{Study Design}

This multicenter, observational, prospective cohort study was conducted at 15 Spanish centers with the involvement of Medical Oncology, Radiation Oncology, and Nutrition departments. The study adhered to the tenets of the Declaration of Helsinki and was approved by the Ethics Committee of Comunidad Autónoma de Aragón (CEICA, Code CP-CI.PI 15/0319). All participants provided written informed consent before inclusion in the study.

The nutritional status of patients, stool characteristics, and other clinical variables were collected before the initiation of nutritional treatment with OEN (baseline) and after 8 weeks. Nutritional support consisted of Survimed OPD Drink ${ }^{\circledR}$ (Fresenius Kabi, Bad Homburg, Germany), a peptide-based sip feed with the following composition: hydrolyzed proteins (18.6\%, whey proteins), carbohydrates (56.4\%: 30\% saccharose and 70\% oligosaccharides), and fat content $(25 \% ; 51 \%$ as medium-chain triglycerides) at a final caloric density of $1 \mathrm{Kcal} / \mathrm{mL}$ (Supplementary Information).

The nutritional protocol comprised the following steps: (1) Assessing the nutritional status of patients with the Subjective Global Assessment (SGA), and (2) Evaluating the intestinal function of patients [13] (see Graphical Abstract). Patients received 2 or 3 bottles per day ( $200 \mathrm{~mL} / \mathrm{bottle})$ of OEN according to their nutritional status and intestinal function.

The OEN protocol was started at the onset of OTRD. Furthermore, patients were recommended to follow an astringent diet (low in insoluble fiber and fat, free of lactose, irritating or flatulent foods). Only patients with watery stools received loperamide at baseline until the consistency improved to mushy. 


\subsection{Study Population}

Patients were included according to the following criteria: adult patients with confirmed diagnosis of cancer, receiving cancer therapy (antitarget therapy, chemotherapy, radiotherapy, or chemotherapy and radiotherapy), and with malnourishment or at risk of malnourishment associated with OTRD.

Patients were excluded from the study when they did not provide written informed consent, their life expectancy was $<3$ months, diarrhea was secondary to the treatment with antibiotics, H2-receptor antagonists or prokinetics, laxatives or osmotically active agents, patients with Clostridium difficile infection, and with any concomitant gastrointestinal disorder not associated with the tumor or cancer treatment.

\subsection{Study Outcomes}

This study aimed to assess the effectiveness of the proposed OEN protocol in improving the nutritional status and stool frequency and consistency in patients with OTRD.

The nutritional status was assessed at baseline and following 8 weeks of OEN treatment through the SGA [15]. The SGA is a nutritional assessment method that considers body weight, body mass index (BMI), dietary intake, gastrointestinal symptoms, physical examination (loss of subcutaneous fat, muscle wasting, etc.), muscular function, and albumin levels. Patients were classified as: (1) at risk of malnourishment, (2) moderate malnourished or (3) severe malnourished. Based on inclusion criteria, all the patients included had some level of malnourishment, and none was well-nourished, being the category "at risk of malnutrition" the best possible clinical status in these patients. We defined that the protocol was effective when: (1) the nutritional status was maintained at risk of malnourishment, (2) improved from moderate to at risk of malnourishment or from severe to moderate malnourishment (1-level improvement), or (3) improved from severe to at risk of malnourishment (2-level improvement). Worsening of nutritional status was considered when: (1) the nutritional status worsened from at risk to moderate malnourishment or from moderate to severe malnourishment (1-level worsening) or (2) from low risk to severe malnourishment (2-level worsening).

Stool consistency was evaluated with the Bristol Stool form scale [16] at baseline and after 8 weeks of OEN treatment. The Bristol Stool form scale classifies the consistency of stools into 7 types; types 1-4 are considered "normal" and types 5 (soft), 6 (mushy) and 7 (watery) indicate diarrhea. The treatment was considered effective when it (1) allowed the maintenance of normal stools (types 1-4); (2) improved stool consistency by 1 level (from 7 to 6,6 to 5 , or 5 to $1-4$ types), 2 levels (from 7 to 5 or 6 to $1-4$ types), or 3 levels (from 7 to 1-4 types). Conversely, we considered that stool consistency worsened when stool classification increased by 1 level (from 1-4 to 5, 5 to 6 , or 6 to 7 type) or 2 levels (from 1-4 to 6 or 5 to 7 ).

Stool frequency was evaluated by recording the number of stools per day at baseline and after 8 weeks of OEN treatment. Given the wide inter-patient variability, stool frequency was not categorized and was then expressed as the mean number of stools/day.

Patients reported the average OEN volume consumed throughout the study and compliance was categorized into the following groups: consumption of all ( $200 \mathrm{~mL} / \mathrm{bottle})$ the OEN content prescribed, 2/3 (150 mL/bottle) of the content prescribed, and 1/2 (100 mL/bottle) of the content prescribed.

Tolerance to the OEN treatment was monitored by recording the frequency $2 \mathrm{~h}$ after the OEN intake of the following events: nausea, vomiting, reflux, abdominal pain, flatulence, satiety, constipation, or stomach heaviness. Patients rated the frequency of each event as "never", "rarely", "sometimes", "often", and "always" after 8 weeks of OEN treatment.

\subsection{Statistical Analyses}

The statistical package IBM SPSS Statistics v.24.0 (IBM Corp, Armonk, NY, USA) was used for statistical analysis. Normal distribution of the data was tested with the Kolmogorov-Shapiro-Wilks test and normality plots. Categorical variables were reported as counts and percentages. Continuous variables were reported as mean and standard deviations. Differences between periods in categorical 
variables were estimated using the McNemar test. Continuous variables were compared across the 2 periods using paired $t$-tests. A $p<0.05$ was considered statistically significant.

Sample size calculations were performed with the McNemar formula for 2 proportions with paired data. Considering a nutritional improvement in $20 \%$ of the population and, assuming a $5 \%$ type I $(\alpha)$ error, $90 \%$ power, and $20 \%$ drop out rate, 145 patients were required for this study.

\section{Results}

\subsection{Study Population}

A total of 162 patients with OTRD who initiated treatment with OEN were included and 149 completed the study. None of the patients received previous enteral nutrition.Mean \pm SD age in the overall population was $68.6 \pm 12.6$ years, and $55 \%$ were men. The tumor was localized in $52.9 \%$ of cases, and $50.4 \%$ of patients received curative treatment, and chemotherapy + radiotherapy was the treatment of choice in $46.3 \%$ of cases (Table 1 ). No patient required a reduction in cancer therapy throughout the study.

Table 1. Demographic and clinical characteristics of study participants.

\begin{tabular}{|c|c|}
\hline & $n=149$ \\
\hline Age (years), Mean (SD) & $68.6(12.6)$ \\
\hline Median (min; max) & $69(30 ; 92)$ \\
\hline \multicolumn{2}{|l|}{ Gender, $(\%)$} \\
\hline Men & $82(55.0 \%)$ \\
\hline Woman & $67(45.0 \%)$ \\
\hline Weight (kg), Mean (SD) & $62.9(12.1)$ \\
\hline BMI $\left(\mathrm{kg} / \mathrm{m}^{2}\right)$, Mean (SD) & $22.6(3.9)$ \\
\hline \multicolumn{2}{|l|}{ Resectability $(* n=136)$} \\
\hline Unresectable & $64(47.1 \%)$ \\
\hline Localized & $72(52.9 \%)$ \\
\hline \multicolumn{2}{|l|}{ Type of treatment $\left({ }^{*} n=131\right)$} \\
\hline Palliative & $65(49.6 \%)$ \\
\hline Curative & $66(50.4 \%)$ \\
\hline \multicolumn{2}{|l|}{ Treatment modality } \\
\hline Chemotherapy & $56(37.6 \%)$ \\
\hline Radiotherapy & $24(16.1 \%)$ \\
\hline Chemotherapy + Radiotherapy & $69(46.3 \%)$ \\
\hline \multicolumn{2}{|l|}{ Targeted therapy } \\
\hline Yes & $28(18.8 \%)$ \\
\hline No & $121(81.2 \%)$ \\
\hline \multicolumn{2}{|l|}{ Cytotoxic treatment } \\
\hline Yes & $99(66.4 \%)$ \\
\hline No & $50(33.6 \%)$ \\
\hline \multicolumn{2}{|l|}{ Type of tumor } \\
\hline Gynecologic/urologic & $29(19.5 \%)$ \\
\hline Colon & $78(52.3 \%)$ \\
\hline Esophagogastric & $22(14.8 \%)$ \\
\hline Other & $20(13.4 \%)$ \\
\hline
\end{tabular}

* number of patients with available data. \% are calculated over the patients with available data. BMI, Body Mass Index. Demographic and clinical characteristics were not significantly different by sex, except for the proportion of specific types of tumors (such as gynecologic).

\subsection{Nutritional Status}

The nutritional status at baseline assessed with the SGA showed that $34.9 \%$ of patients were at risk of malnourishment (lowest level of malnourishment), 54.4\% were moderately malnourished, and 10.7\% severely malnourished. After 8 of weeks of nutritional support with OEN, the proportion of patients at risk of malnourishment increased $(62.2 \%)$ while that of moderately and severely malnourished decreased (34.5\% and 3.4\%, respectively). The changes in the nutritional status from baseline to week 8 were statistically significant $(p<0.001)$. This resulted in an improvement in the nutritional status in 
$48.3 \%$ of patients and a worsening in $14.1 \%$ of patients. Taken together, OEN support was effective in $68.5 \%$ of cases: $48.3 \%$ improved the nutritional status, and $20.1 \%$ were maintained at low risk of malnourishment (the best possible status in these patients) (Figure 1).

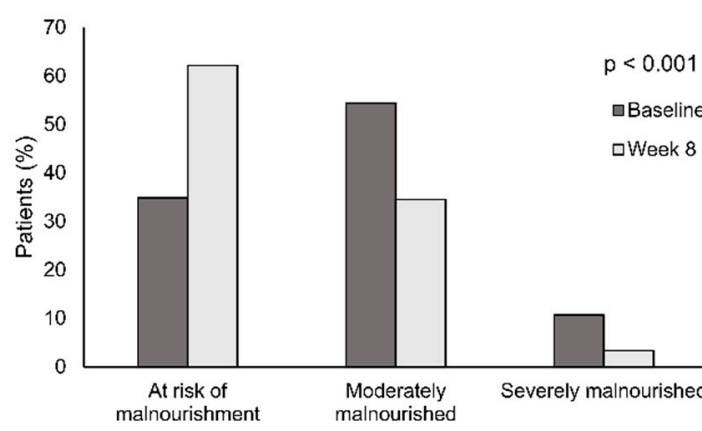

\begin{tabular}{cl}
\hline Manteinance nutritional status & $56(37.6 \%)$ \\
Maintenance low-risk malnutrition & $30(20.1 \%)$ \\
Maintenance moderate malnutrition & $26(17.4 \%)$ \\
Maintenance severe malnutrition & 0 \\
Improvement nutritional status & $72(48.3 \%)$ \\
1-level improvement & $57(38.3 \%)$ \\
2-level improvement & $15(10.1 \%)$ \\
Worsening nutritional status & $21(14.1 \%)$ \\
1-level worsening & $19(12.8 \%)$ \\
2-level worsening & $2(1.3 \%)$ \\
Effectiveness & $102(68.5 \%)$ \\
\hline
\end{tabular}

Figure 1. Change in nutritional status after 8 weeks of oligomeric enteral nutrition (OEN). The bars show the proportion of patients at risk of malnourishment and with moderate or severe malnourishment at baseline and after 8 weeks of OEN treatment. The change in the nutritional status from baseline to week 8 was statistically significant $(p<0.001)$. The table shows the changes in the nutritional status (improvement and worsening) and the effectiveness of the OEN algorithm in improving the nutritional status. Patient's nutritional status was classified as: (1) at risk of malnourishment, (2) moderate malnourishment, or (3) severe malnourishment.

The nutritional status significantly improved in all patient subgroups regardless of the type of tumor, type of treatment, treatment modality, resectability, and whether they received targeted therapy or cytotoxic treatment (Figure S1).

BMI was significantly higher following 8 weeks of OEN treatment compared to baseline (22.93 vs. $22.64 \mathrm{~kg} / \mathrm{m}^{2} ; p=0.004$ ) (Figure 2). Serum albumin $(\mathrm{g} / \mathrm{dL})$ levels were $2.88 \mathrm{~g} / \mathrm{dL}$ at baseline and significantly increased with the OEN protocol after 8 weeks $(3.45 \mathrm{~g} / \mathrm{dL} ; p<0.001)$ (Figure 2$)$. Differences in the change in BMI did not reach statistical significance for patients receiving radiotherapy, radiotherapy + chemotherapy, targeted therapies, or non-cytotoxic therapy. The change in albumin levels was statistically significant for all the patient subgroups.

\subsection{Stool Consistency and Frequency}

Stool consistency significantly changed with the 8-week OEN protocol $(p<0.001)$. The proportion of patients with normal and soft stools increased (from $14.1 \%$ to $33.6 \%$ and from $21.5 \%$ to $37.6 \%$ ), whereas mushy and watery stools decreased (from $38.3 \%$ to $26.8 \%$ and from 26.2 to $2 \%$ ) (Figure 3). Changes in stool consistency from baseline to week 8 were statistically significant $(p<0.001)$. After 8 weeks of nutritional support with OEN, $57.7 \%$ of patients changed the stool consistency, with $24.2 \%$ and $24.8 \%$ showing 1- and 2-level improvement, respectively. The treatment showed a $71.1 \%$ effectiveness in improving stool consistency (13.4\% maintained stool types $1-4$ and $57.7 \%$ improved stool consistency). 
Stool consistency was significantly better after 8 weeks of OEN treatment in all stratification groups, except for the subgroups of patients receiving targeted therapy or non-cytotoxic treatment.
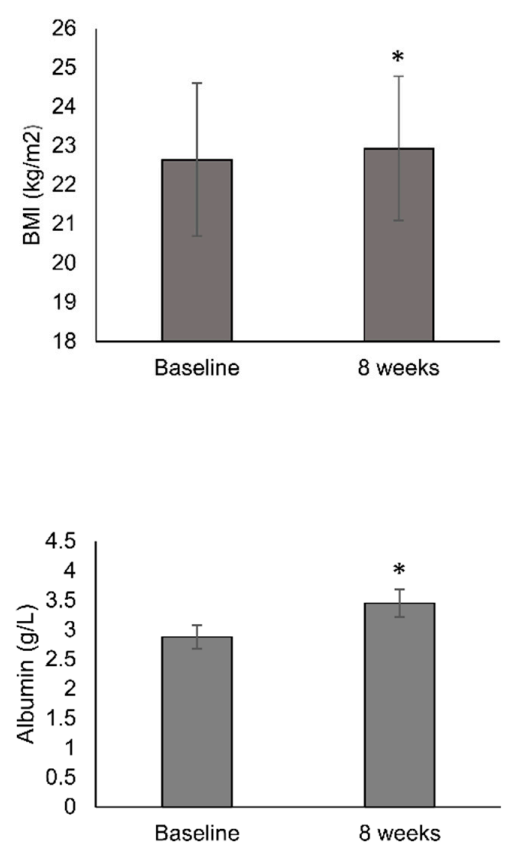

Figure 2. Change in body mass index and albumin levels after 8 weeks of oligomeric enteral nutrition (OEN). The bars show mean \pm SD levels for body mass index (BMI) and albumin at baseline and after 8 weeks of OEN treatment. * indicates $p<0.05$ (from baseline to week 8).

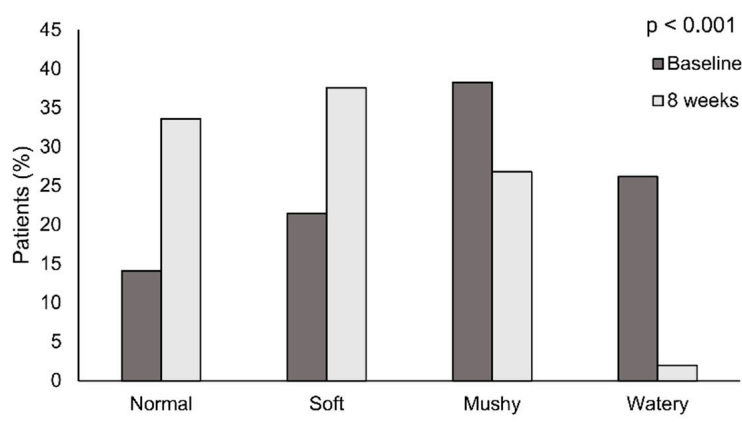

\begin{tabular}{cl}
\hline Maintenance stool consistency & $39(26.2 \%)$ \\
Maintenance types 1-4 (normal) & $20(13.4 \%)$ \\
Maintenance type 5 (soft) & $13(8.73 \%)$ \\
Maintenance type 6 (mushy) & $6(4.03 \%)$ \\
Maintenance type 7 (watery) & $0(0 \%)$ \\
Improvement stool consistency & $86(57.7 \%)$ \\
1-level improvement & $36(24.2 \%)$ \\
2-level improvement & $37(24.8 \%)$ \\
3-level improvement & $13(8.7 \%)$ \\
Worsening stool consistency & $24(16.1 \%)$ \\
1-level worsening & $17(11.4 \%)$ \\
2-level worsening & $7(4.7 \%)$ \\
Effectiveness & $106(71.1 \%)$ \\
\hline
\end{tabular}

Figure 3. Change in stool consistency after 8 weeks of oligomeric enteral nutrition (OEN). The bars show the proportion of normal, soft, mushy, and watery stools at baseline and after 8 weeks of OEN treatment. Changes in stool consistency from baseline to week 8 were statistically significant $(p<0.001)$. The table shows the proportion of patients with changes in stool consistency and the effectiveness of the OEN protocol in improving stool consistency. 
The mean number of stools per day decreased from baseline $(4.17 \pm 3.33$ stools/day) to week 8 $(1.42 \pm 1.63$ stools/day; $p=0.0041)$ (Figure 4). Stool frequency significantly improved in all stratification groups with the exception of patients that received targeted therapy.

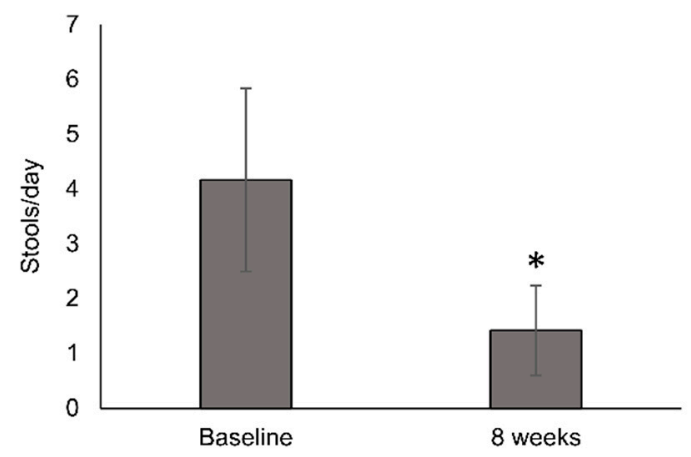

Figure 4. Change in stool frequency after 8 weeks of oligomeric enteral nutrition (OEN) treatment. The bars show mean \pm SD stools/day at baseline and after 8 weeks of OEN treatment. The change in stool frequency from baseline to week 8 was statistically significant $(p=0.0041)$. ${ }^{*}$ mean the statistical significance $(p=0.0041)$.

Cross-tabulation of nutritional status levels and stool characteristics showed that the change in nutritional status did not depend on the change in stool consistency ( $p=0.34$; Chi-square).

\subsection{Patients with Persistent Diarrhea}

We next analyzed the subgroup of patients for whom the presence of diarrhea did not improve with the OEN protocol. Despite not improving the consistency or frequency of stools, we observed a significant improvement in the nutritional status $(p=0.04)$ and albumin levels $(p=0.009)$ in those patients with persistent diarrhea. Of note, $46 \%$ of patients improved the nutritional status $(40 \%$ by 1 level and $6 \%$ by 2 levels). In those patients with persistent diarrhea, the nutritional status worsened in $16 \%$ of patients and was maintained in $38 \%$ (with $12 \%$ of patients at risk of malnourishment and $26 \%$ with moderate malnourishment) (Figure 5).

\subsection{Compliance}

$72.3 \%$ of patients reported consuming the total content of OEN prescribed, $20.9 \% 2 / 3$ of the content prescribed and $6.8 \% \frac{1}{2}$ of the content prescribed throughout the study. Nutritional support with OEN was associated with good tolerability in $80.3 \%$ of patients and with moderate intolerance in $19.7 \%$ of patients (Table 2).

Table 2. Compliance and tolerance to oligomeric enteral nutrition (OEN) after 8 weeks of treatment.

\begin{tabular}{cc}
\hline Treatment Compliance $\left.{ }^{*} \boldsymbol{n}=\mathbf{1 4 8}\right)$ & \\
\hline Total content prescribed & $107(72.3 \%)$ \\
$2 / 3$ bottle content prescribed & $31(20.9 \%)$ \\
$1 / 2$ bottle content prescribed & $10(6.8 \%)$ \\
Treatment tolerance $\left({ }^{*} n=147\right)$ & \\
Good tolerance & $118(80.3 \%)$ \\
Moderate intolerance & $29(19.7 \%)$ \\
Severe intolerance & $0(0.0 \%)$ \\
\hline
\end{tabular}

* Number of patients with available data. \% are calculated over the patients with available data. 


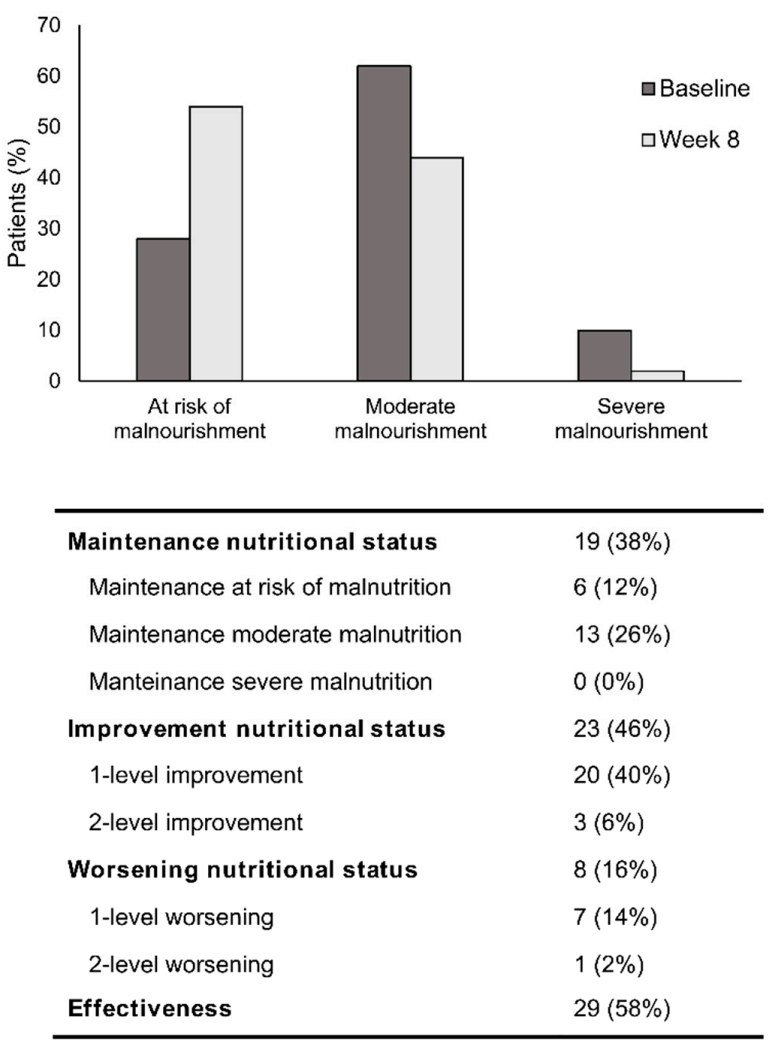

Figure 5. Nutritional status evolution in those 50 patients who did not improve diarrhea after 8 weeks of oligomeric enteral nutrition (OEN). The graph shows the proportion of patients at risk of malnourishment, with moderate or severe malnourishment at baseline and after 8 weeks of nutritional support with OEN. The table shows the proportion of patients with changes in the nutritional status and the effectiveness of the OEN protocol in improving the nutritional status in patients with persistent diarrhea.

When analyzing compliance rates in different patient subgroups, we observed that compliance rates were significantly higher after 8 weeks of OEN treatment relative to baseline in all subgroups. Nevertheless, we found poorer compliance rates in patients under palliative vs. curative care and with unresectable vs. localized tumors. Regarding the modality of cancer treatment, compliance was lower in patients receiving chemotherapy vs. radiotherapy or both, targeted vs. non-targeted therapy, or cytotoxic vs. non-cytotoxic treatment.

The majority of patients rated the frequency of different symptoms as "never" or "rarely" present. Regarding potential symptoms associated with OEN treatment, the reported absence of symptoms was the following: no nausea in $82.3 \%$ of patients, no vomiting in $88.4 \%$ of patients, no reflux in $78.2 \%$ of patients, no flatulence in $69.2 \%$ of patients, no abdominal pain in $77.8 \%$ of patients, no satiety in $51.7 \%$ of patients, no constipation in $91.2 \%$ of patients, and no heaviness in $68 \%$ of patients (Figure S2).

\section{Discussion}

This study showed that the proposed OEN algorithm seemed effective in improving the nutritional status, frequency and consistency of stools and was associated with high compliance rates in patients with OTRD.

Unlike previous studies, we included 149 oncologic patients with several types of tumors and receiving different cancer treatment modalities. The overall analysis of patients allowed us to assess the general response to OEN in OTRD patients, whereas patient subgroups were useful for assessing specific responses. In the overall population, mean age was 68.6 years, most of the patients included 
were at risk of malnourishment or moderately malnourished (88.4\%), and the most common tumor type was colon.

An important finding of our study is the improvement in the nutritional status observed in $48.3 \%$ of patients and the effectiveness of the treatment (68.5\%). We observed an increase in the proportion of patients at risk of malnourishment and a decrease in those with moderate/severe malnourishment in the overall population and in different patient subgroups in our study. Unlike other studies using the turnover rate of plasma proteins (prealbumin, transferrin, and retinol-binding protein) and changes in body weight as indicators of nutritional status, this study comprehensively assessed the nutritional status of patients through the SGA, BMI change, and albumin levels. Indeed, to our knowledge, none of the studies using elemental diet in cancer patients used the SGA to assess the nutritional status, despite the fact that it is considered the recommended nutritional assessment test for cancer patients. The randomized controlled trial comparing the efficacy of oligomeric vs. polymeric formulas used the Prognostic Nutritional Index (PNI) and the Controlling Nutritional Status (CONUT) and showed significantly better scores for the CONUT in patients receiving the oligomeric formula [17]. Most of the studies using elemental diet formulations reported no significant differences in the nutritional status between intervention and control groups in cancer patients [18-21]. In contrast, in one study the elemental diet improved the intestinal mucosal inflammation and nutritional status of patients with Crohn's Disease [14]. However, it is important to note that previous results were based on well-nourished patients at baseline, and in our study, all the patients included had certain level of malnourishment.

In line with the improvement in nutritional status revealed by the SGA, serum albumin levels significantly increased from 2.88 to $3.45 \mathrm{~g} / \mathrm{dL}$ upon treatment with OEN. A previous study reported a decrease in albumin after 4 weeks of Elental ${ }^{\circledR}$ nutritional support ( $80 \mathrm{~g} / 300$ kcal amino-acid-rich, fat free, elemental diet) [22], while in other study the changes were minimal and non-significant after 6 months of either oligomeric or polymeric formula [17] or 14 days of microbial immune nutrition [23].

OEN was also associated in our study with a significant increase in BMI after 8 weeks. Previous results reporting the effect of nutritional support on BMI have been heterogeneous. The effect of Elental $^{\circledR}$ on body weight was inconsistent, with one randomized study reporting a significant difference in body weight change when compared with the control group in patients who received adjuvant chemotherapy for gastric cancer [24], other study showing a preservation of lean body mass after 4 weeks of nutritional support during chemo- or chemoradiotherapy in patients with esophageal cancer [22], and two studies showing no differences in patients with oral squamous cell carcinoma [18,19]. Furthermore, Ohkura et al. found no significant differences in the change in body weight between oligomeric and polymeric formulas after esophagectomy [17]. The importance of body weight improvement is derived from the association between this variable and negative quality of life and worse long-term prognosis [25] and also considering that severe weight loss (>10\%) has been acknowledged as a risk factor for non-relapse mortality [26].

The OEN protocol was effective in improving stool consistency, with $57.7 \%$ of patients showing an improvement, and a remarkable reduction in the proportion of watery stools (from $25.5 \%$ to $2 \%$ ). These improvements resulted in the OEN treatment being effective for stool consistency in $71.1 \%$ of cases. In analyzing these results, it is important to consider that patients with watery stools at baseline received loperamide until changing the consistency to mushy. Although the use of this antidiarrheal drug contributed to improving the consistency in those patients, its use in only $26.2 \%$ of patients does not fully explain the improvement in stool consistency observed in the overall population (57.7\%). Likewise, the reduction in stool frequency from 4.17 stools/day at baseline to 1.42 stools/day after 8 weeks was also striking. Regarding cancer therapy modality, the higher stool frequency per day at baseline was observed in patients receiving chemotherapy and radiotherapy (5.46 stools/day) as previously reported [8], although this subgroup also experienced the highest decrease in stool frequency after OEN (reduction of -4.13 stools/day). In this context, it is interesting to note that the subgroup of patients receiving both chemotherapy and radiotherapy also showed the highest 
compliance. Regarding previously reported effects of enteral nutrition on diarrhea, a study found that the frequency of diarrhea was significantly lower with an oligomeric vs. a polymeric formula [17], and two showed a higher incidence of diarrhea in the elemental diet group as compared with the control group [27] or azulene oral rinse [22].

A relevant finding of this study is that the OEN support seemed effective in improving the nutritional status even in those patients with persistent diarrhea despite the OEN protocol. This result is of remarkable interest since it implies that patients could benefit from this OEN protocol, despite the fact that they do not notice substantial changes in the frequency and consistency of stools.

Patients reported high compliance rates with the OEN support, with $72.3 \%$ reporting ingesting the total content prescribed. The high compliance rates observed are in line with the good tolerability $(80.3 \%)$ associated with this nutritional support. Importantly, common symptoms responsible for enteral nutrition interruptions [12], such as nausea or vomiting, were seldom present in our study. Satiety, heaviness and flatulence were the symptoms with a higher incidence, although still in $>80 \%$ of cases were "never" or "rarely" reported. These results are of particular importance, as poor tolerability and palatability of oral nutritional formulas, together with tolerability symptoms associated with cancer treatment, are the main barriers to treatment compliance that lead to suboptimal effectiveness of nutritional supports [28]. Previous studies provided heterogeneous results regarding treatment compliance. In patients receiving chemotherapy and Elental ${ }^{\circledR}$ formula as elemental diet, a compliance rate of $71.4 \%$ was reported for patients with gastric cancer [24] and of 70\% in patients with esophageal cancer [29]. Poorer compliance rates were observed in patients undergoing pelvic radiotherapy and partial replacement with oral E028, showing a median overall daily intake of $21 \%$ of caloric requirements [21].

Taken together, we observed that OEN seemed to serve its first purpose of improving the nutritional status of patients. One of the main contributors to this effectiveness could be the compliance with the treatment that allows the optimal effect of this nutritional support. Treatment compliance, in turn, is likely influenced by the improvement in health status (nutritional, body weight, frequency of stools), which further encourages patients to remain compliant. The improved nutritional status is also probably reflecting the higher digestibility of a peptide-based formulation, which has the potential to maintain the gastrointestinal mucosa integrity and facilitate nutrient absorption [29]. Furthermore, oligomeric formulas have been associated with reduced mucosal production of pro-inflammatory cytokines [14] and pancreatic or biliary stimulation [30]. It is important to highlight that we cannot directly compare our results with those using elemental supplements previously published given their different methodological design [13]. First, most of the studies included a limited number of patients $[22,29,31]$. Second, the nutritional doses provided were different (in most of the studies patients received $300 \mathrm{Kcal}$ and $14.1 \mathrm{~g} /$ protein/day and, in our study, patients received between $400 \mathrm{Kcal}$ and $18.6 \mathrm{~g} /$ protein/day and $600 \mathrm{Kcal}$ and $27.9 \mathrm{~g} /$ protein/day) [18,29,32]. Third, the follow-up period of these studies was relatively short for achieving nutritional changes [21,22]. Fourth, none of the studies primarily aimed to assess the impact of nutritional supports on the nutritional status and, in several of them, this was neither a secondary endpoint [31]. Fifth, when reported, patients were well-nourished at baseline, so better results are expected in patients with certain level of malnourishment as in our study $[18,19,22,24]$.

There are different limitations associated with this study that are worth mentioning. First, the lack of control group does not allow to rule out that factors, other than the OEN protocol, could have conditioned the results obtained. Potential factors could be following an astringent diet in all patients, the use of transient antidiarrheal drugs in patients with watery stools until changing the consistency to mushy, or the evolution of diarrhea itself. Further investigations with a controlled design are needed to confirm our results. However, as this is the first study following the proposed OEN protocol in clinical practice, our study was not intended to compare the effectiveness of this protocol with other nutritional support but to test whether the protocol help improve the nutritional status. In this regard, since this observational study included patients with nutritional deficit for whom the absence of nutritional 
support could have compromised their status, the use of placebo was not considered. Moreover, as previous animal studies reported reduced pancreatic or biliary stimulation with oligomeric vs. polymeric diet [30] and a lower frequency of diarrhea and improved nutritional status was observed with a oligomeric vs. a polymeric formula [17], we did not consider comparing the effectiveness of our OEN protocol with a polymeric formula. Second, the inclusion of patients did not follow selective inclusion criteria. Although this allowed us to obtain comprehensive information among a wide range of clinical groups, the sample size in some subgroups was not powered to establish reliable conclusions. Third, the duration of follow up was relatively short to capture long term differences. Fourth, we cannot directly compare the compliance rates obtained in our study with those previously observed, as patients reported the total volume ingested throughout the study and other studies were based on the doses recorded in the patient diary. Fifth, antidiarrheal drugs were used only in patients with watery stools until the consistency improved to mushy. As the proportion of patients who reported watery stools at baseline was $26.2 \%$, the use of loperamide does not completely explain the improvement in the frequency and consistency of stools observed in the overall population. Although loperamide undoubtedly contributed to improving the consistency of stools, we observe this effect also in patients not treated with this antidiarrheal drug (soft or mushy stools consistency, 59.8\% of patients). Finally, due to the observational design of our study, we cannot ensure that the clinical benefits observed are fully due to the OEN protocol.

In contrast, this study is one of the first comprehensively describing the effectiveness of an OEN protocol targeting both the nutritional status of patients and the evolution of OTRD. Our study included a considerable sample size, which is larger than that in most of the studies published to date and assessed the nutritional status with a valid and reliable tool [33,34] and with other nutritional indicators (albumin, BMI). To our knowledge, this study is the first in assessing the change in nutritional status with an OEN protocol in patients with different types of tumors. Moreover, most of the previous studies were conducted in Japan [17-20,22,24,27,29,31], and the present study provides valuable information for patients in Europe.

\section{Conclusions}

The OEN protocol proposed in this study seemed effective in improving patient's nutritional status, stool frequency, and consistency with high rates of compliance in patients with OTRD.

Supplementary Materials: The following are available online at http://www.mdpi.com/2072-6643/12/5/1534/s1, Figure S1: Change in nutritional status after 8 weeks of oligomeric enteral nutrition (OEN) support by patient subgroups, Figure S2: Frequency of tolerability symptoms after 8 weeks of oligomeric enteral nutrition (OEN).

Author Contributions: Conception of the work: A.S.-P.; design of the nutritional decision algorithm: A.S.-P.; F.C.-G., J.M.-T., and J.L.-S.; acquisition data: A.S.-P.; F.C.-G., J.M.-T., and J.L.-S. and DIAPOENO Study group; analysis and interpretation of data for the work: A.S.-P. and R.M.-V.; drafting of the work and critical revision for important intellectual content: A.S.-P. and R.M.-V; funding acquisition: A.S.-P. All authors have read and agreed to the published version of the manuscript.

Funding: Miguel Servet Biomedical Foundation.

Acknowledgments: The following investigators participated in the acquisition data of the OEN study (multi-author group): Eduardo Polo (Oncology department, Miguel Servet, Zaragoza); Marta Marcos (Oncology Department, Hosp San Juan, Alicante); Antonio Blanco Orones (Geriatrics department, H. Virgen del Valle, Toledo); Juan Ignacio Delgado Mingorance (Oncology department, Infanta Cristina, Badajoz); Jesús José Cuadrado Blanco (Geriatrics department, Residencia Diputación Salamanca); Berta Jimenez (Oncology department, Hosp Ernest Lluch, Calatayud); Daniel Antonio de Luis Román (Clínico Valladolid); Olatz Izaola Jauregui (Dietitian, Clínico Valladolid, Valladolid); Radiation Oncology department (Hospital Universitario Ramón y Cajal, Fundación Investigación Biomédica); Rodolfo Augusto Chicas Sett (Hospital Campo Grande, Valladolid); Miguel Angel Berenguer (Oncology department, Hosp San Juan, Alicante); Laura Cerezo (Oncology department, Hospital La Princesa, Madrid); Ana Isabel Ferrer (Oncology department, Obispo Polanco, Teruel); Ana I. Alonso García (Hosp Central de Asturias, Asturias); Raul Amurrio Quevedo (H. Rey Juan carlos, Móstoles); M. Dolores de las Peñas Cabrera (H. Rey Juan carlos, Móstoles). Carla Granados from Trialance SCCL provided medical writing support. We want to thank the Miguel Servet Biomedical Foundation for the financial support provided for the publication of this article. 
Conflicts of Interest: A. Sanz-Paris and Fernando Calvo-Gracia have received speaker's fees and has worked on research projects from Abbott Nutrition, Fresenius-Kabi, Nestlé, and Nutricia. Javier Martinez-Trufero and Julio Lambea-Sorrosal have received speaker's fees and worked on research projects from Fresenius-Kabi, Abbott, Pharmamar, Eisai, Merck, Bristol-Myers Squibb, Angelini and Lilly. The funders had no role in the design of the study; in the collection, analyses, or interpretation of data; in the writing of the manuscript; or in the decision to publish the results.

\section{References}

1. Elting, L.S.; Cooksley, C.; Chambers, M.; Cantor, S.B.; Manzullo, E.; Rubenstein, E.B. The burdens of cancer therapy: Clinical and economic outcomes of chemotherapy-induced mucositis. Cancer 2003, 98, 1531-1539. [CrossRef] [PubMed]

2. Grant, M.; Kravits, K. Symptoms and their impact on nutrition. Semin. Oncol. Nurs. 2000, 16, $113-121$. [CrossRef] [PubMed]

3. Richardson, G.; Dobish, R. Chemotherapy induced diarrhea. J. Oncol. Pharm. Pract. 2007, 13, 181-198. [CrossRef] [PubMed]

4. Andreyev, J.; Ross, P.; Donnellan, C.; Lennan, E.; Leonard, P.; Waters, C.; Wedlake, L.; Bridgewater, J.; Glynne-Jones, R.; Allum, W.; et al. Guidance on the management of diarrhoea during cancer chemotherapy. Lancet Oncol. 2014, 15, e447-e460. [CrossRef]

5. Arends, J.; Bachmann, P.; Baracos, V.; Barthelemy, N.; Bertz, H.; Bozzetti, F.; Fearon, K.; Hütterer, E.; Isenring, E.; Kaasa, S.; et al. ESPEN guidelines on nutrition in cancer patients. Clin. Nutr. 2017, 36, 11-48. [CrossRef]

6. Thompson, K.L.; Elliott, L.; Fuchs-Tarlovsky, V.; Levin, R.M.; Voss, A.C.; Piemonte, T. Oncology Evidence-Based Nutrition Practice Guideline for Adults. J. Acad. Nutr. Diet. 2017, 117, 297-310. [CrossRef]

7. Dranitsaris, G.; Maroun, J.; Shah, A. Estimating the cost of illness in colorectal cancer patients who were hospitalized for severe chemotherapy-induced diarrhea. Can. J. Gastroenterol. 2005, 19, 83-87. [CrossRef]

8. Benson, A.B.; Ajani, J.A.; Catalano, R.B.; Engelking, C.; Kornblau, S.M.; Martenson, J.A.; McCallum, R.; Mitchell, E.P.; O'Dorisio, T.M.; Vokes, E.E.; et al. Recommended guidelines for the treatment of cancer treatment-induced diarrhea. J. Clin. Oncol. 2004, 22, 2918-2926. [CrossRef] [PubMed]

9. Peterson, D.E.; Boers-Doets, C.B.; Bensadoun, R.J.; Herrstedt, J. Management of oral and gastrointestinal mucosal injury: ESMO Clinical Practice Guidelines for diagnosis, treatment, and follow-up. Ann. Oncol. 2015, 26, v139-v151. [CrossRef]

10. Wedlake, L.J.; Shaw, C.; Whelan, K.; Andreyev, H.J.N. Systematic review: The efficacy of nutritional interventions to counteract acute gastrointestinal toxicity during therapeutic pelvic radiotherapy. Aliment. Pharmacol. Ther. 2013, 37, 1046-1056. [CrossRef]

11. Cederholm, T.; Barazzoni, R.; Austin, P.; Ballmer, P.; Biolo, G.; Bischoff, S.C.; Compher, C.; Correia, I.; Higashiguchi, T.; Holst, M.; et al. ESPEN guidelines on definitions and terminology of clinical nutrition. Clin. Nutr. 2017, 36, 49-64. [CrossRef] [PubMed]

12. Chen, Y.; Peterson, S.J. Enteral Nutrition Formulas: Which Formula Is Right for Your Adult Patient? Nutr. Clin. Pract. 2009, 24, 344-355. [CrossRef] [PubMed]

13. Sanz-Paris, A.; Martinez-García, M.; Martinez-Trufero, J.; Lambea-Sorrosal, J.; Calvo-Gracia, F.; López-Alaminos, M.E. Oligomeric enteral nutrition in undernutrition, due to oncology treatment-related diarrhea. Systematic review and proposal of an algorithm of action. Nutrients 2019, 11, 1888. [CrossRef] [PubMed]

14. Yamamoto, T.; Nakahigashi, M.; Umegae, S.; Kitagawa, T.; Matsumoto, K. Impact of Elemental Diet on Mucosal Inflammation in Patients with Active Crohn's Disease: Cytokine Production and Endoscopic and Histological Findings. Inflamm. Bowel Dis. 2005, 11, 580-588. [CrossRef] [PubMed]

15. Detsky, A.; Baker, J.M.; Johnston, N.; Whittaker, S.; Mendelson, R.; Jeejeebhoy, K. What is subjective global assessment of nutritional status? J. Parenter. Enter. Nutr. 1987, 11, 8-13. [CrossRef]

16. Lewis, S.J.; Heaton, K.W. Stool Form Scale as a Useful Guide to Intestinal Transit Time. Scand. J. Gastroenterol. 1997, 32, 920-924. [CrossRef] 
17. Ohkura, Y.; Ueno, M.; Shindoh, J.; Iizuka, T.; Udagawa, H. Randomized controlled trial on efficacy of oligomeric formula (HINE E-GEL ${ }^{\circledR}$ ) versus polymeric formula $\left(\right.$ MEIN $^{\circledR}$ ) enteral nutrition after esophagectomy for esophageal cancer with gastric tube reconstruction. Dis. Esophagus 2019, 32, 1-10. [CrossRef]

18. Harada, K.; Ferdous, T.; Horinaga, D.; Uchida, K.; Mano, T.; Mishima, K.; Park, S.; Hanazawa, H.; Takahashi, S.; Okita, A.; et al. Efficacy of elemental diet on prevention for chemoradiotherapy-induced oral mucositis in patients with oral squamous cell carcinoma. Support. Care Cancer 2016, 24, 953-959. [CrossRef]

19. Harada, K.; Minami, H.; Ferdous, T.; Kato, Y.; Umeda, H.; Horinaga, D.; Uchida, K.; Park, S.; Hanazawa, H.; Takahashi, S.; et al. The Elental ${ }^{\circledR}$ elemental diet for chemoradiotherapy-induced oral mucositis: A prospective study in patients with oral squamous cell carcinoma. Mol. Clin. Oncol. 2018, 159-167. [CrossRef]

20. Imamura, H.; Nishikawa, K.; Kishi, K.; Inoue, K.; Matsuyama, J.; Akamaru, Y.; Kimura, Y.; Tamura, S.; Kawabata, R.; Kawada, J.; et al. Effects of an Oral Elemental Nutritional Supplement on Post-gastrectomy Body Weight Loss in Gastric Cancer Patients: A Randomized Controlled Clinical Trial. Ann. Surg. Oncol. 2016, 23, 2928-2935. [CrossRef]

21. Mcgough, C.; Wedlake, L.; Baldwin, C.; Hackett, C.; Norman, A.R.; Blake, P.; Harrington, K.; Tait, D.; Khoo, V.; Frost, G.; et al. Clinical trial: Normal diet vs. partial replacement with oral E028 formula for the prevention of gastrointestinal toxicity in cancer patients undergoing pelvic radiotherapy. Aliment. Pharmacol. Ther. 2008, 27, 1132-1139. [CrossRef] [PubMed]

22. Ishikawa, T.; Yasuda, T.; Doi, T.; Okayama, T.; Sakamoto, N.; Gen, Y.; Dohi, O.; Yoshida, N.; Kamada, K.; Uchiyama, K.; et al. The amino acid-rich elemental diet Elental ${ }^{\circledR}$ preserves lean body mass during chemo- or chemoradiotherapy for esophageal cancer. Oncol. Rep. 2016, 36, 1093-1100. [CrossRef] [PubMed]

23. Shao, F.; Xin, F.; Yang, C.; Yang, D.; Mi, Y.; Yu, J.; Li, G. The Impact of Microbial Immune Enteral Nutrition on the Patients with Acute Radiation Enteritis in Bowel Function and Immune Status. Cell Biochem. Biophys. 2014, 69, 357-361. [CrossRef] [PubMed]

24. Toyomasu, Y.; Mochiki, E.; Yanai, M.; Suzuki, M.; Yanoma, T.; Kimura, A.; Kogure, N.; Ogata, K.; Kuwano, H. A prospective pilot study of an elemental nutritional supplement for prevention of oral mucositis during S-1 adjuvant chemotherapy for gastric cancer. Surg. Oncol. 2019, 29, 97-101. [CrossRef]

25. Kong, H.; Kwon, O.K.; Yu, W. Changes of quality of life after gastric cancer surgery. J. Gastric Cancer 2012, 12, 194-200. [CrossRef]

26. Fuji, S.; Mori, T.; Khattry, N.; Cheng, J.; Do, Y.R.; Yakushijin, K.; Kohashi, S.; Fukuda, T.; Kim, S.W. Severe weight loss in 3 months after allogeneic hematopoietic SCT was associated with an increased risk of subsequent non-relapse mortality. Bone Marrow Transp. 2015, 50, 100-105. [CrossRef]

27. Morishita, T.; Tsushita, N.; Imai, K.; Sakai, T.; Miyao, K.; Sakemura, R.; Kato, T.; Niimi, K.; Ono, Y.; Sawa, M. The Efficacy of an Oral Elemental Diet in Patients Undergoing Hematopoietic Stem Cell Transplantation. Intern. Med. 2016, 55, 3561-3569. [CrossRef]

28. Wall, C.L.; Gearry, R.B.; Day, A.S. Polymeric formula is more palatable than elemental formula to adults with Crohn's disease. e-SPEN J. 2014, 9, e200-e203. [CrossRef]

29. Tanaka, Y.; Ueno, T.; Yoshida, N.; Akutsu, Y.; Takeuchi, H.; Baba, H.; Matsubara, H.; Kitagawa, Y.; Yoshida, K. The effect of an elemental diet on oral mucositis of esophageal cancer patients treated with DCF chemotherapy: A multi-center prospective feasibility study (EPOC study). Esophagus 2018, 15, 239-248. [CrossRef]

30. O'Keefe, S.J.D.; Lee, R.B.; Anderson, F.P.; Gennings, C.; Abou-Assi, S.; Clore, J.; Heuman, D.; Chey, W. Physiological effects of enteral and parenteral feeding on pancreaticobiliary secretion in humans. Am. J. Physiol. Liver Physiol. 2003, 284, G27-G36. [CrossRef]

31. Ogata, Y.; Ishibashi, N.; Yamaguchi, K.; Uchida, S.; Kamei, H.; Nakayama, G.; Hirakawa, H.; Tanigawa, M.; Akagi, Y. Preventive effects of amino-acid-rich elemental diet Elental ${ }^{\circledR}$ on chemotherapy-induced oral mucositis in patients with colorectal cancer: A prospective pilot study. Support. Care Cancer 2016, 24, 783-789. [CrossRef] [PubMed]

32. Morita, T.; Fujimoto, K.; Shima, Y.; Ogata, A.; Kumanogoh, A. Minimal neonatal transfer of certolizumab pegol in a Japanese patient with rheumatoid arthritis. Ann. Rheum. Dis. 2018, 77, 22-24. [CrossRef] [PubMed] 
33. Laky, B.; Janda, M.; Cleghorn, G.; Obermair, A. Comparison of different nutritional assessments and body-composition measurements in detecting malnutrition among gynecologic cancer patients. Am. J. Clin. Nutr. 2008, 87, 1678-1685. [CrossRef]

34. Bauer, J.; Capra, S.; Ferguson, M. Use of the scored Patient-Generated Subjective Global Assessment (PG-SGA) as a nutrition assessment tool in patients with cancer. Eur. J. Clin. Nutr. 2002, 56, 779-785. [CrossRef] [PubMed]

(C) 2020 by the authors. Licensee MDPI, Basel, Switzerland. This article is an open access article distributed under the terms and conditions of the Creative Commons Attribution (CC BY) license (http://creativecommons.org/licenses/by/4.0/). 